Dhaka Univ. J. Sci. 65(1) 35-39, 2017 (January)

\title{
Effect of Birth Interval on Neonatal and Post Neonatal Mortality in Bangladesh
}

\author{
RadiaTaisir*, Most. Fatima-Tuz-Zahura and Wasimul Bari \\ Department of Statistics, Biostatistics and Informatics, Dhaka University, Dhaka-1000, Bangladesh
}

(Received: 13 March 2016; Accepted: 28 August 2016)

\begin{abstract}
This study investigates the relationship between previous birth interval and infant mortality using the data extracted from the Bangladesh Demographic and Health Survey (BDHS), 2011. For the purpose of regression analysis, the Cox proportional hazard model has been used. Results demonstrate significant effects of previous birth interval on neonatal mortality, but not on post neonatal mortality. If the previous birth interval is less than two years or greater than three years, the index child experiences high risk of neonatal mortality.
\end{abstract}

Key Words: Birth Interval, Log-rank Test, Neonatal Mortality, Post Neonatal Mortality, Proportional Hazard Model

\section{Introduction}

Despite having strong association between birth interval and infant mortality, it has been received less attention by the policy makers at the public health sector of developing countries like Bangladesh. Infant mortality rate plays an important role to reflect the overall health scenario of a country. Therefore, it is essential to quantify the mechanism by which birth interval might reduce the infant mortality as well as improve the child health. On the other hand, infancy period (first 364 days of a child) can be divided into two segments: neonatal period (first 28 days) and post neonatal period (29 to 364 days), where first segment is considered as the most critical period for a child. To reduce under-five mortality by two-thirds, especially in low and middle income countries, between 1990 and 2015, 189 United Nation member states agreed to implement Millennium Development Goal 4. Still now approximately 10,000 newborns experience death everyday during the neonatal period and up to $40 \%$ of under-five mortality is attributed to the neonatal death ${ }^{14}$. In this paper, it is of interest to study how the preceding birth interval having influence on the infant mortality acts on the neonatal and post neonatal mortality in Bangladesh.

Many studies have already demonstrated the importance of birth spacing for the survival chances of infants. By analyzing the world fertility survey data, Hobcraft et al. ${ }^{11}$ found a strong evidence for increased mortality risk among children born after short birth interval. Ronsmans ${ }^{19}$ examined the association between birth spacing and child mortality by using logistic and Cox proportional hazards regression model. Curtis et al. ${ }^{7}$ found highly significant effect of preceding birth interval on post neonatal mortality in Brazil by using random-effects logistic models. Boerma et al. ${ }^{4}$ used the Demographic and Health Surveys (DHS) data to investigate the pathways through which preceding birth intervals may affect child survival. Their study revealed the association between preceding birth interval and neonatal mortality, mortality at 1-6 months of age and 7-23 months of age. Using the data from Bangladesh and the Philippines, Miller et al. ${ }^{17}$ demonstrated that children who are born within 15 months of preceding birth are 60 to 80 percent more likely to die than that of other children in their first two years of life. Koenig et al. ${ }^{16}$ used a longitudinal data from Bangladesh and found significant effect of preceding birth interval on neonatal mortality but the effect was substantially less than that found in other studies. Conde-Agudelo et al. ${ }^{5}$ found the association among both long and short intervals with other adverse maternal outcome of pregnancy. DaVanzo et al. ${ }^{8}$ found that the women whose pregnancies occurred between 15 and 75 months after a preceding pregnancy outcome have a lower likelihood of fetal loss than those with shorter or longer inter-pregnancy interval by using data from Matlab demographic surveillance system in Bangladesh. He also found that the shorter intervals are associated with higher mortality after controlling for other correlates of infant and child mortality ${ }^{9}$. Hosseinpoor et al. ${ }^{12}$ argued that a birth interval less than 24 months birth-to-birth with an adjusted odds ratio of 2.22 contributed 13 percent of inequality in infant mortality between births as measured by concentration index. Akmatov et al. ${ }^{1}$ revealed that birth intervals of 18 months or longer have adjusted odd ratio of 0.61 for mortality of children under 36 months after controlling for 12 socioeconomic, bio-demographic, reproductive health care etc. Rizviet al. ${ }^{18}$ showed that the children are more likely to be healthy and to survive through all developmental stages upto age five years who born after a three-year birth interval. While a birth interval of less than 18 months was associated with increased risk for neonatal mortality (3.17 times), infant mortality (3.16 times) and under-five mortality (2.81 times) as compared to a $36-47$ month birth interval. Emily ${ }^{10}$ found the length of interpregnancy interval as a significant contributor to neonatal mortality from a population-based retrospective cohort study. On the basis of a modified conceptual framework for child survival, using hierarchical modeling,Kayode ${ }^{14}$ found that neonates with inadequate birth spacing had a lower chance of surviving. Bhalotraet al. ${ }^{3}$ found that birth intervals explain only a partial fraction of the correlation between neonatal mortality of successive children in a family using dynamic panel data in India. Saha et. al. ${ }^{20}$ analyzed the causal effects of birth spacing on subsequent infant mortality.

In this paper, data extracted from Bangladesh DHS (BDHS), 2011 has been analyzed using Product-Limit approach and log-rank test for bivariate analysis and Cox proportional hazard model for the regression analysis. This paper is organized in four sections. In section 2, data and methodology are discussed briefly. Results obtained from bivariate analysis and regression models are given in Section 3.Finally, the paper is concluded in section 4. 


\section{Data and Methodology}

Data

This study used data extracted from the Bangladesh Demographic and Health Survey (BDHS), 2011. Following a two-stage stratified sampling design, the survey selected 600 enumeration areas (EAs) with probability proportional to EA size at the first stage and the second stage involved selecting 30 householdsfrom each of the selected EA's that comprises a total of 17,141 households. Of the households, BDHS finally interviewed successfully 17,842 ever married women aged 12-49 years on a complete history of their live births, including the sex, month and year of each birth, survival status and age at the time of the survey and age at death along with socio-economic and demographic variables. To get information for this study, first we considered all children who were born preceding five years of survey, and then children with birth order number one were excludet to calculate the previous birth interval. For neonatal mortality, information from 5602 children has been collected, out of those 149 have died before surviving their $28^{\text {th }}$ day of life that emerges the neonatal mortality rate 26.60 per thousand live births.

For the analysis of post neonatal mortality, the sample consisted of 5385 children surviving after 28 days. Out of those, 62 have died within 364 days resulting post neonatal mortality rate 11.51 per thousand live births. These rates for both neonatal and post neonatal mortality slightly underestimate the rates given in the BDHS 2011 report. This is because this study excludes children, who died within the neonatal and post neonatal period, but they were born before preceding 5 years of the survey.

\section{Variables}

The outcome variable in this study is the time to death within 28 days for the neonatal mortality and within 29 to 364 days for post neonatal mortality. A child was considered to be censored if he/she did not die within neonatal and post neonatal period and the censoring time for this child were taken as 28 days and 364 days for neonatal and post neonatal mortality, respectively. The key independent variable is the length of the previous birth interval, measured as the number of months between the date of birth of the index child and the immediately preceding birth to the mother. It is expected that the duration of 2-3 years between two successive births might reduce the adverse effect of maternal and child health outcomes ${ }^{21}$. This leads to the choice of three categories of variable previous birth interval: 0 to 24 months, 25-36 months, and more than 36 months. It is found that $14.42 \%$ of children considered for the neonatal mortality was born within 2 years of their older sibling, $19.64 \%$ within 25-36 months and $65.94 \%$ was born in a long duration after their immediate previous sibling. These percentages for post neonatal mortality are $14.20 \%, 19.90 \%$, and $65.90 \%$.There are a number of factors that may potentially confound the relationship between birth intervals and young child mortality. Based on the literatures mentioned earlier, factors include birth specific confounders and mother specific confounders. The variables mother's age at birth, maternal education, region, wealth index, place of residence have been considered in this study as mother specific confounder. Birth specific confounders include birth order, sex of child, size of child, preceding sibling's survival status, yearof birth, season of birth and place of delivery.

\section{Statistical Methods}

The study examines the unadjusted and adjusted effects of birth interval on neonatal and post neonatal mortality using survival and hazard functions, respectively. Product-Limit approach ${ }^{13}$ is used to estimate the survival functions under different categories of selected covariates and log-rank test ${ }^{15}$ has been used to examine whether there exists any significant differences among the survival functions. To find out the adjusted effect of covariates semi parametric proportional hazard $(\mathrm{PH})$ regression model $^{6}$ is used. Note that covariates that were found to have significant unadjusted effect are only considered in the regression model.

\section{Results}

\section{Survival Analysis Results}

The main interest of this section is to investigate the existence of unadjusted effects of previous birth interval on neonatal and post neonatal mortality. The survival experiences for different categories of previous birth interval have been plotted in Figure 1 and 2 along with p-value obtained from log rank test for neonatal and post neonatal mortality, respectively.

In the case of neonatal mortality, the risk of mortality is highest for the children born within 24 months of a previous birth. Babies born within 25-36 months of its previous birth experience the lowest risk of mortality than the other two categories. For post neonatal mortality, it is observed from Figure 2 that initially survival probability is highest for category 25-36 months and it increases with the increase of previous birth interval. For both cases, differences are significant as p-values are less than 0.05. Table 1 shows the logrank test $\mathrm{p}$-values for other selected variables under neonatal and post neonatal mortality. 


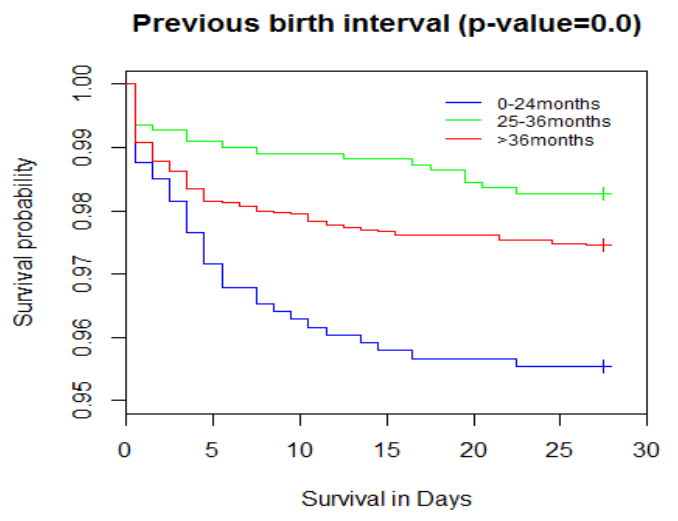

Fig. 1. Survival curves by previous birth interval for neonatal mortality.
Previous birth interval ( $p$-value $=0.045)$

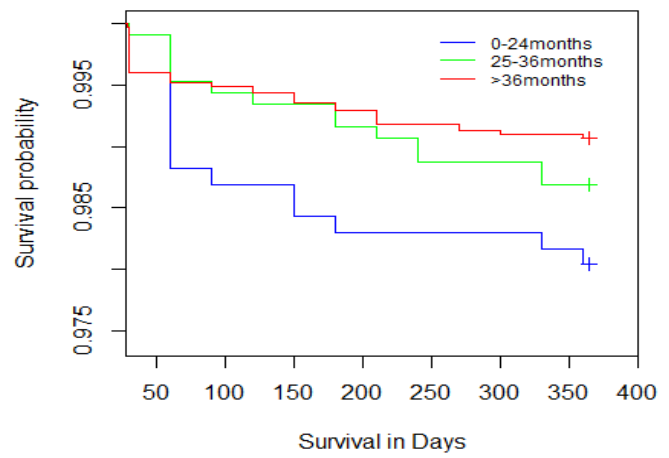

Fig. 2. Survival curves by previous birth interval for post neonatal mortality.

Table 1. Log-rank test p-values for the selected variables under neonatal and post-neonatal mortality

\begin{tabular}{lcc}
\hline \multicolumn{1}{c}{ Variables } & \multicolumn{2}{c}{ Log-rank test p-values } \\
\cline { 2 - 3 } & Neonatal mortality & Post neonatal mortality \\
\hline Mother's age at birth & 0.105 & 0.183 \\
Region & 0.019 & 0.310 \\
Maternal education & 0.020 & 0.001 \\
Wealth index & 0.945 & 0.083 \\
Birth order & 0.076 & 0.001 \\
Sex of child & 0.136 & 0.127 \\
Place of residence & 0.861 & 0.861 \\
Size of child & 0.000 & 0.121 \\
Preceding sibling's survival status & 0.001 & 0.000 \\
Year of birth & 0.524 & 0.574 \\
Season of birth & 0.402 & 0.262 \\
Place of delivery & 0.008 & 0.061 \\
\hline
\end{tabular}

The above table confirms that the variables region, maternal education, birth order, previous birth interval, size of child, preceding sibling's survival status and place of delivery have significant relationship with neonatal mortality. On the other hand, significant relationships have been found between post neonatal mortality and the variables maternal education, wealth index, birth order, previous birth interval, preceding sibling's survival status and place of delivery.

Further analysis have been carried out using Cox proportional hazard model in next subsection to assess the adjusted effect of the previous birth interval on neonatal and post neonatal mortality controlling for the effects of those covariates that were found significant from log-rank test $\mathrm{p}$ values.

\section{Survival Regression Results}

Table 2 presents the hazard ratios and $\mathrm{p}$-values for the considered covariates under infant, neonatal and post neonatal mortalities obtained from the proportional hazard model.

\section{Infant Mortality}

It is clear from the table that controlling for other covariates, occurrence of infant mortality is significantly less if the previous birth interval is over 2 years. The hazard rate for infant mortality is $47.1 \%$ less for the birth interval $25-36$ months and $29.9 \%$ less if the birth interval is above 3 years compared to the birth interval less than or equal to 2 years. Higher education of mother is found to be a significant factor for reducing the infant mortality. In other education levels, mortality rate remains same in Bangladesh. Among the region, the hazard rate for infant mortality is significantly lowest in Khulna and it is $53.9 \%$ lower compared to Dhaka. If preceding sibling of the index child is alive, the chance of dying for the index child decreases significantly and the hazard rate is $50.6 \%$ lower compared to an index child whose preceding child is dead. The size of child at birth is also an important factor for infant mortality as hazard rate is $43.5 \%$ less for a child if his/her size at birth is average or large compared to a child with small size at birth. Mother's age at birth is not found as risk factor for infant mortality and wealth index, birth order of index child, and place delivery are not considered in the model as these are not associated significantly with the infant mortality.

\section{Neonatal mortality}

Effect of previous birth interval on neonatal mortality is similar to the effect found for the infant mortality. A child is at the highest risk of dying within 28 days of life, if he/she 
was born within 2 years of his/her preceding sibling's birth. The hazard rates for neonatal mortality are $57.1 \%$ and $34.3 \%$ lower if the previous birth interval is 25-36 months and greater than 3 years, respectively compared to birth interval 2 years or less. In addition, maternal education, region, preceding sibling's survival status, birth order, size of child and place of delivery have been found as potential risk factors in analyzing the neonatal mortality data. Children of higher educated mothers have $65.6 \%$ less hazard rate of dying within 28 days than those of illiterate mothers. A significant difference in neonatal mortality has been observed in region. In this case, the analysis reveals that children born to mothers residing in Chittagong and Khulna division have significantly $45.1 \%$ and $55.8 \%$ lower hazard rate compared to the children living in Dhaka with p-values 0.054 and 0.044 , respectively. Hazard rate for neonatal morality is significantly 1.748 times for children with an older sibling who died compared to the children having older sibling alive ( $\mathrm{p}$-value 0.00). Moreover, a child who is the fourth or higher in order has significantly $35 \%$ lower hazard rate for neonatal mortality than the child with order second or third ( $\mathrm{p}$-value=0.04). Results also show that the child having a size average or large at the time of birth has significantly $42.5 \%$ less hazard to die $(p$-value $=0.002)$ than those born with a small size.It is surprising to notice that the rate of dying is $87.9 \%$ higher if the index child was born at hospital/clinic compared to the child born at home or other places and this finding is found statistically significant at $1 \%$ level of significance ( $\mathrm{p}$-value 0.001).This may happen because in developing country like Bangladesh, pregnant women often go to hospital or clinic if severe complexities arise during the time of delivery.

\section{Post neonatal mortality}

Though previous birth interval plays an important role on infant mortality as well as neonatal mortality, it has no significant impact on the post natal mortality. To examine the effect of education, secondary and higher education levels were considered together as few observations were found in higher education level. Table 2 confirms that children from secondary or higher educated mothers have $53.1 \%$ less hazard rate for post neonatal mortality than those from illiterate mothers and the result is significant $(\mathrm{p}$ value $=0.051$ ). Moreover, a child whose older sibling died is 2.80 times as likely as to die than a child whose older sibling is alive and this result is statistically significant (pvalue $=0.001$ ). Wealth index, birth order, and place of delivery, on the other hand, do not seem to affect postneonatal mortality to a large extent.

Table 2.Adjusted hazard ratio (HR) obtained from Cox PH model for neonatal, post neonatal and infant mortality for selected socio-economic and demographic variables

\begin{tabular}{|c|c|c|c|c|c|c|c|}
\hline \multirow[b]{3}{*}{ Covariate } & \multirow[b]{3}{*}{ Category } & \multicolumn{6}{|c|}{ Mortality } \\
\hline & & \multicolumn{2}{|c|}{ Infant } & \multicolumn{2}{|c|}{ Neonatal } & \multicolumn{2}{|c|}{ Post Neonatal } \\
\hline & & HR & p-value & HR & p-value & HR & p-value \\
\hline \multirow{3}{*}{$\begin{array}{l}\text { Previous birth } \\
\text { interval }\end{array}$} & $0-24(\mathrm{RC})$ & - & - & - & - & - & - \\
\hline & $25-36$ & 0.529 & 0.005 & 0.429 & 0.003 & 0.715 & 0.369 \\
\hline & $36+$ & 0.701 & 0.053 & 0.657 & 0.044 & 0.632 & 0.154 \\
\hline \multirow{3}{*}{$\begin{array}{l}\text { Mother's age at } \\
\text { birth (years) }\end{array}$} & $<20$ & 1.342 & 0.123 & & & & \\
\hline & $20-30$ (RC) & - & - & $\mathrm{NC}$ & & $\mathrm{NC}$ & \\
\hline & $30+$ & 1.181 & 0.350 & & & & \\
\hline \multirow{4}{*}{$\begin{array}{l}\text { Maternal } \\
\text { education }\end{array}$} & No education(RC) & - & - & - & - & - & - \\
\hline & Primary & 1.169 & 0.362 & 1.431 & 0.103 & 0.644 & 0.147 \\
\hline & Secondary & 0.839 & 0.357 & 0.802 & 0.380 & $0.469^{*}$ & 0.051 \\
\hline & Higher & 0.386 & 0.066 & 0.344 & 0.054 & & \\
\hline \multirow[t]{7}{*}{ Region } & Barisal & 1.124 & 0.658 & 1.023 & 0.932 & & \\
\hline & Chittagong & 0.764 & 0.268 & 0.549 & 0.054 & & \\
\hline & Dhaka (RC) & - & - & - & & & \\
\hline & Khulna & 0.461 & 0.040 & 0.442 & 0.044 & $\mathrm{NC}$ & \\
\hline & Rajshahi & 1.116 & 0.664 & 0.898 & 0.717 & & \\
\hline & Rangpur & 1.157 & 0.564 & 0.979 & 0.943 & & \\
\hline & Sylhet & 1.193 & 0.429 & 1.273 & 0.341 & & \\
\hline \multirow[t]{3}{*}{ Wealth Index } & Poor & & & & & 1.339 & 0.466 \\
\hline & Middle (RC) & $\mathrm{NC}$ & & $\mathrm{NC}$ & & & \\
\hline & Rich & & & & & 1.365 & 0.476 \\
\hline \multirow{2}{*}{$\begin{array}{l}\text { Preceding } \\
\text { sibling's } \\
\text { survival status }\end{array}$} & Dead (RC) & - & - & - & - & - & - \\
\hline & Alive & 0.494 & 0.000 & 0.572 & 0.012 & 0.357 & 0.001 \\
\hline \multirow{2}{*}{$\begin{array}{l}\text { Birth order } \\
\text { number }\end{array}$} & $2^{\text {nd }}-3^{\text {rd }}(\mathrm{RC})$ & & & - & - & - & - \\
\hline & $4^{\text {th }}+$ & $\mathrm{NC}$ & & 0.650 & 0.040 & 1.559 & 0.105 \\
\hline \multirow{2}{*}{$\begin{array}{l}\text { Size of child at } \\
\text { birth }\end{array}$} & Small (RC) & - & - & - & - & $\mathrm{NC}$ & \\
\hline & Average/ large & 0.565 & 0.002 & 0.575 & 0.002 & & \\
\hline \multirow[t]{2}{*}{ Place of delivery } & Hospital/clinic & $\mathrm{NC}$ & & 1.879 & 0.001 & 0.661 & 0.335 \\
\hline & Others (RC) & & & - & - & - & \\
\hline
\end{tabular}

*This category was created by combining secondary and higher since cell frequency in higher group is too small; NC: Not considered in model; RC: Reference category 


\section{Conclusion}

Neonatal period is the most vulnerable stage of a child's life to survive and from this study it is found that $70.6 \%$ of the total death occurred in the infancy period is covered up by the neonatal stage. Using bivariate (KM curve and log-rank test) and multivariate techniques (Cox's proportional hazard regression), the effect of previous birth interval has been studied on infant mortality, broken down into two periods: neonatal and post neonatal. The magnitude of the risk of infant mortality associated with previous birth interval is significantly large and almost explained by the neonatal period. The effect of previous birth interval on infant mortality obtained from survival regression model is found very similar to those for neonatal mortality. It is observed that previous birth interval affects neonatal mortality to a large extent after controlling for other important birthspecific and mother-specific confounders. But it is surprising to notice that deaths occurred during postneonatal period are not affected by previous birth interval.This study reveals the fact that too short and too long previous birth interval are potential risk factors for the neonatal mortality. A child born within two years after a preceding birth outcome experiences the highest risk of neonatal mortality.It is also found that a child born with a previous birth interval greater than 3 years is also at higher risk of neonatal mortality compared to a child with previous birth interval between 2 and 3 years.

As being one of the most important determinants of early childhood death, attention to control the adverse effect of previous birth interval needs to be surged. To reduce the infant mortality as well as the neonatal mortality to a great extent, public health policy makers should provide the optimal birth spacing for parents. The findings of the current study suggest that parents who want their children to survive and thrive would do well to wait at least 24 months and also not to wait too long after a birth to have another child.

In this paper, it was assumed that birth intervals obtained from children are independent. It was found in the data considered in the analysis that some birth intervals were resulted from the same mother. Such birth intervals were correlated and this correlation needs to be taken into account for the purpose of estimation to obtain more precise estimates of parameters of interest. This is the main limitation of this paper.

\section{References}

1. Akmatov, M. K., R. T. Mikolajczyk \& A.Krämer, 2006. Determinants of neonatal and under-three mortality in Central Asian countries: Kyrgyzstan, Kazakhstan and Uzbekistan. GMS Med InformBiomEpidemiol. 2(2), Doc08.

2. Bangladesh Demographic and Health Survey (BDHS), 2011. NIPORT, Dhaka, Bangladesh; Mitra and Associates, Dhaka/ Bangladesh.

3. Bhalotra, S. \& A. V. Soest, 2008. Birth-spacing, Fertility and Neonatal Mortality in India: Dynamics, Frailty and Fecundity. Journal of Econometrics. 143, 274-290.
4. Boerma, J. T. \& T. G.Bicego,1992. Preceding Birth Intervals and Child Survival: Searching for Pathways of Influence. Stud famplann, 23, 243-56.

5. Conde-Agudelo, A., A.Rosas-Bermu'dez \& A. C.KafuryGoeta, 2007. Effects of birth spacing on maternal health: a systematic review. American Journal of Obstetrics \& Gynecology. 196(4),.297-308.

6. Cox, D. R., 1972. Regression Models and Life-tables (with discussion).Journal of the Royal Statistical Society, Series B, 34, 187-220.

7. Curtis, S. L., I.Diamond, \& J. W.McDonald, 1993. Birth Interval and Family Effects on Postneonatal Mortality in Brazil. Demography, 30, (1), 33-43.

8. DaVanzo, J., L. Hale, A. Razzaque \& M.Rahman, 2007. Effects of interpregnancy interval and outcome of the preceding pregnancy on pregnancy outcomes in Matlab, Bangladesh. BJOG: An International Journal of Obstetrics \&Gynaecology. 114(9), 1079-1087.

9. DaVanzo, J., L. Hale, A.Razzaque \& M.Rahman, 2008. The Effects of Pregnancy Spacing on Infant and Child Mortality in Matlab. Bangladesh: How They Vary by The Type of Pregnancy Outcome That Began The Interval. Population Studies: A Journal of Demography. 62(2), 131-154.

10. Emily, A., DeFranco, L. M.Seske, J.M. Greenberg \& L. J. Muglia, 2015. Influence of Interpregnancy Interval on Neonatal Morbidity. American Journal of Obstetrics \& Gynecology. 212, 386.e1-9.

11. Hobcraft, J. N., J. W. McDonald \& S.O.Rutstein, 1985. Demographic Determinants of Infant and early child mortality: A comparative analysis. Popul Stud, 39, 363-85.

12. Hosseinpoor, A.R., E. V.Doorslaer, N. Speybroeck, M. Naghavi, K. Mohammad, R. Majdzadeh, B. Delavar, H. Jamshidi \& J. Vega, 2006. Decomposing socioeconomic inequality in infant mortality in Iran. International Journal of Epidemiology.35(5), 1211-1219.

13. Kaplan, E. L. and P. Meier, 1958. Nonparametric Estimation from Incomplete Observations, J. Amer. Statis.Ass., 53, 457-481.

14. Kayode, G. A., E. Ansah, I. A. Agyepong, M. AmoakohColeman, D. E. Grobbee\&K. Klipstein-Grobusch, 2014. Individual and Community Determinants of Neonatal Mortality in Ghana: a Multilevel Study. BMC Pregnancy and Childbirth. 14(165), 1471-2393.

15. Kleinbaum, D.G. \& M. Klein, 2005. Survival Analysis: A Selflearning Text, second edition, Springer-Verlag, New York.

16. Koenig, M. A., J. F. Phillips, O. M. Campbell \& S. D’Souza, 1990. Birth Intervals and Chilhood Mortality in Rural Bangladesh. Demography. 27, 251-65.

17. Miller, J. E., J. Trussel, A. R. Pebley \& B. Vaughan, 1992.Birth Spacing and Child Mortality in Bangladesh and the Philippines. Demography. 29(2), 305-318.

18. Rizvi, F. \& A. Khan, 2011. Birth Spacing as a Health Intervention. Ann. Pak. Inst. Med. Sci., 7(3), 113-114.

19. Ronsmans, C., 1996. Birth Spacing and Child Survival in Rural Senegal. International Journal of Epidemiology, 25(5), 989-97.

20. Saha, U. R. \& A. V. Soest, 2013. Contraceptive Use, Birth Spacing and Child Survival in Matlab, Bangladesh. Studies in Family Planning. 44(1), 45-66.

21. World Health Organization, 2005. Report of a technical consultation on birth spacing. Geneva, Switzerland. 
Dhaka Univ. J. Sci. 65(1) 35-39, 2017 (January) 